\title{
Predictive masking is associated with a system-wide reconfiguration of neural populations in the human visual cortex
}

\author{
Joana Carvalho*, Remco J. Renken ${ }^{+}$, Frans W. Cornelissen* \\ *Laboratory of Experimental Ophthalmology, University Medical Center Groningen, University of \\ Groningen, Groningen, the Netherlands \\ ${ }^{+}$Cognitive Neuroscience Center, University Medical Center Groningen, University of Groningen, \\ Groningen, the Netherlands
}

Correspondence: Joana Carvalho, Laboratory of Experimental Ophthalmology, University Medical Center Groningen, University of Groningen, P.O. Box 196, 9700 AD, Groningen, the Netherlands. Phone: +31 50 3616965. E-mail: j.c.de.oliveira.carvalho@rug.nl

Conflict of Interest: None of the authors has potential conflicts of interest to be disclosed.

Keywords: prediction, masking, population receptive field, connective field, reorganization, artificial scotoma, fMRI 


\section{Abstract}

2 The human visual system masks the perceptual consequences of retinal or cortical lesion-induced

3 scotomas by predicting what is missing from nearby regions of the visual field. To reveal the

4 neural mechanisms underlying this remarkable capacity, known as predictive masking, we used

5 fMRI and neural modeling to track changes in cortical population receptive fields (pRFs) and

6 connectivity in response to the introduction of an artificial scotoma (AS). Consistent with

7 predictive masking, we found that extrastriate areas increased their sampling of the V1 region

8 outside the AS projection zone. Moreover, throughout the visual field and hierarchy, pRFs

9 shifted their preferred position towards the AS border. A gain field model, centered at this

10 border, accounted for these shifts, especially for extrastriate areas. This suggests that a system-

11 wide reconfiguration of neural populations in response to a change in visual input is guided by

12 extrastriate signals and underlies the predictive masking of scotomas. 


\section{Introduction}

14 When the information extracted from a visual scene is incomplete, the visual system attempts to

15 predict what is missing based on information from nearby regions of the visual field. A

16 remarkable perceptual consequence is the masking of retinal lesions, which makes patients

17 remain unaware of their partial loss of vision. Consequently, such masking often results in

18 delayed diagnosis and treatment $(1,2)$ of such lesions. The underlying process to which we will

19 refer to as predictive masking (PM), also plays a prominent role in healthy perception, e.g

20 evident from the masking of the blind spot, the receptorless area of the retina where the optic

21 nerve leaves the eye, and from many visual illusions in which color, brightness, or textures

22 spread into and mask neighbouring regions of the visual field $(3,4)$. Consequently, the process is

23 sometimes also popularly referred to by this behavioral manifestation as "filling-in".

24 Despite the scientific and clinical relevance of PM, its underlying neuronal mechanisms are still

25 poorly understood. Human and animal physiology studies into PM and studies of the neural

26 consequences of retinal lesions have shown receptive field (RF) expansion and shifts in RF

27 preferred position towards spared portions of the visual field (5-9). However, such RF changes

28 also occur following simulated scotomas, thus suggesting that these changes may not result from

29 structural plasticity (10-12). Indeed, the observed RF changes may be an indirect consequence of

30 a modulation in the responses of neurons in the scotoma projection zone (SPZ), possibly caused

31 by gain adjustments that reduce the feedforward information (13-16), a downregulation of

32 inhibition (17), or a change in feedback from higher order areas with large RFs (18-21).

33 Such observations have led to the controversial hypothesis that predictive masking is explained

34 by neurons modifying their receptive field properties, (22) while the precise neural basis of PM 
35 remains unknown. In addition, previous studies assumed that PM is a local process restricted to

36 the SPZ, so they focused on the SPZ and the early visual cortex. However, if PM is a

37 consequence of functional changes (changes in gain), we would expect neurophysiological

38 modifications to occur both inside and outside the SPZ and throughout the visual hierarchy. In

39 the present study, we therefore tested the hypothesis that PM involves a global reconfiguration of

40 RFs and their connectivity. Specifically, in analogy to the behavioral phenomenon, we expect

41 that in the cortical region responsible for PM, the neural mechanisms within the SPZ should

42 show a decreased reliance on information from within the SPZ and an increased reliance on the

43 information from outside of it. If this hypothesis is confirmed, we could create more accurate

44 models of visual perception and improve diagnostic methods for patients with visual field

45 defects.

46 To test our hypothesis, we used functional MRI in combination with biologically-inspired neural

47 population modeling to track changes in RF properties and cortical connectivity following the

48 introduction of an artificial scotoma (AS) into the visual field of human participants (thus

49 mimicking a lesion to their visual system). We modeled the observed changes in pRF preferred

50 position using a gain field model and we examined how cortical connections between recording

51 sites (connective field size) changed in response to the AS.

\section{Results}

53 Retinotopic mapping was performed under three different stimulus conditions: a conventional

54 retinotopy stimulus based on luminance contrast (LCR) used for delineating visual areas, an

55 artificial scotoma stimulus $\left(\mathrm{AS}^{+}\right)$and a control stimulus identical to $\mathrm{AS}^{+}$but without the artificial

56 scotoma $\left(\mathrm{AS}^{-}\right)$. The stimuli used in the two AS conditions were designed to stimulate the Low 
57 Spatial Frequency (LSF) selective neurons predominantly. The LSF carries coarse information

58 about the visual scene and it is presumably encoded mainly by neurons with large RFs $(23,24)$.

59 This is expected to facilitate PM. The $\mathrm{AS}^{-}$and $\mathrm{AS}^{+}$conditions were used to define the pRFs size

60 and preferred position (PP) for each voxel (see materials and methods section for additional

61 details).

\section{The scotoma border attracts $p R F s$}

63 To examine the presence of changes in $\mathrm{pRF}$ properties between the $\mathrm{AS}^{-}$and $\mathrm{AS}^{+}$conditions, the

64 data for the four different quadrants (each containing one AS) was collapsed onto a single

65 quadrant. Next, the pRF properties of the voxels were spatially binned based on their preferred

66 position (PP) as estimated in the $\mathrm{AS}^{-}$condition. In visual area $\mathrm{V} 1$, following the presentation of

67 an AS, pRFs with a PP originally inside the AS shifted radially outwards and towards the border

68 of the AS (Figure 1A). However, an analysis of the entire V1 representation showed that pRFs

69 outside of the AS also appear to be attracted towards the AS (Figure 1B). These shifts were

70 observed across the visual hierarchy (Figure S1 and S2). We compared the PP in both conditions

71 across the visual hierarchy using a two-way repeated measures ANOVA, which revealed main

72 effects of condition $\left(\mathrm{AS}^{-}\right.$versus $\left.\mathrm{AS}^{+}, \mathrm{F}(1,35)=8.4, \mathrm{p}=0.004\right)$ and $\mathrm{ROI}(\mathrm{F}(5,35)=4.09, \mathrm{p}=0.003)$.

73 Furthermore the PP shifts were more pronounced for extrastriate areas (the interaction between

74 ROI and condition was significant $(\mathrm{F}(5,35)=7.87, \mathrm{p}=0.0034)$, see Figure $\mathrm{S} 1)$. Post hoc tests

75 (FDR corrected) showed significant differences in position between conditions for all the visual

76 areas tested $(\mathrm{p}<0.001)$. These observations suggest that $\mathrm{pRF}$ s throughout the visual field shifted

77 their PP towards the AS border. When analyzed in more detail, Figure 1C shows how the PP

78 shifted as a function of the pRFs' distance to the center of the AS. Note that the shift is minimal

79 at the border (at $2.5 \mathrm{deg}$.). Figure D plots the radial component of PP shift, again as a function of 
the pRFs distance to the AS center. This shows a nearly perfect linear relationship between the

81 radial shift and the pRFs' initial $\mathrm{PP}\left(\mathrm{r}^{2}<-0.99\right.$ and $\mathrm{p}<1 \times 10^{-8}$ for all the visual areas, Figure S2).

82 Note that pRFs situated at the AS border hardly shift radially. Additional analyses excluded that

83 these patterns are simply the result of statistical or modeling biases (Figures S3 and S4).
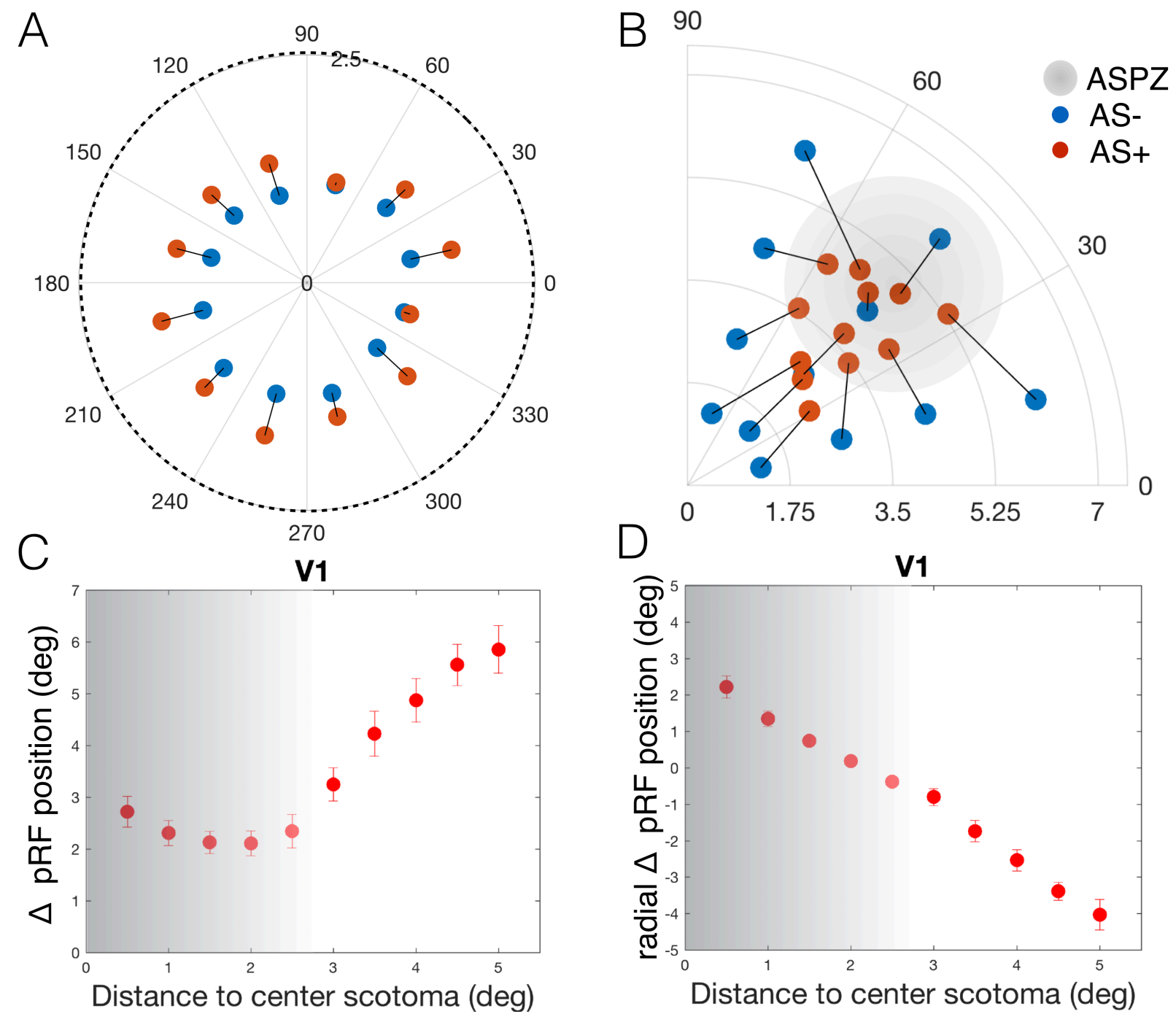

Distance to center scotoma (deg)

Distance to center scotoma (deg)

86 pRFs with initial PPs located inside the ASPZ, averaged across participants. B: Position change between conditions in various

87 sectors of the visual field, averaged across participants. C: pRF position change $\left(\mathrm{AS}^{+} \mathrm{vs} \mathrm{AS}^{-}\right)$as a function of distance between pRF position (based on $\mathrm{AS}^{-}$) and the center of the scotoma (bins of 0.5 deg., Euclidean space). Error bars show the standard error of the mean over hemispheres. D: pRF position change projected onto the radius as a function of the radial distance between $\mathrm{pRF}$ 
corresponds to the center if the AS. Figure S2 shows the results for the other visual areas, V2-LO2. Figure S3 shows that these

92 results are not simply due to random position noise. The $\mathrm{AS}^{+}$results were obtained using the Scotoma Field (SF) model (which

93 minimizes model biases). The pRF position shifts between $\mathrm{AS}^{-}$and $\mathrm{AS}^{+}$were present using either model (SF and FF, Figure $\mathrm{S} 4$ ).

\section{A gain field model explained the artificial scotoma induced $p R F$ position shifts}

95 The systematic changes in pRF PP suggest that these shifts may depend on their position relative

96 to the AS border. Such shifts can be modeled using a gain field (GF) (25). To determine whether

97 the border plays a critical role in the pRF reconfiguration, we first plotted the radial component

98 of the shifts (Figure 2A). This indicates that the shifts are of similar magnitude all around the

99 perimeter of the AS (although different for pRFs initially inside or outside the AS). Next, we

100 determined if we could predict the radial component in the $\mathrm{AS}^{+}$condition based on the PPs in the

$101 \mathrm{AS}^{-}$condition by modulating the AS effect using a GF that is centered on the AS border (Figure

102 5B). Figure 2 shows the predicted and measured pRF positions shifts (Panel 2B) and size ratios

103 (Panel 2C). The GF model performed well and explained 50\% and $92 \%$ of the variance in the

104 radial position shifts and size changes, respectively (Figures 2B and C). Figure 2D shows that

105 the position predictions of the GF model are most accurate for the higher order areas (V1, VE

$106=39 \%$; LO1, VE=66\%). The PP shifts tend to increase along the visual hierarchy (Figure S1).

107 Although the pRF sizes increased with eccentricity and visual hierarchy (Pearson's correlation

108 coefficient: $\mathrm{r}^{2}>0.8$ and $\mathrm{p}<0.05$ for all the visual areas tested), the pRF PP change does not

109 strongly correlate with the pRF size within every visual field map (V1 $r^{2}=0.06 ; \quad V 2 r^{2}=-0.06$;

$110 \mathrm{~V} 3 \mathrm{r}^{2}=0.13 ; \mathrm{V} 4 \mathrm{r}^{2}=-0.06 ; \mathrm{LO} 1 \mathrm{r}^{2}=0.1 ;$ LO2 $\mathrm{r}^{2}=-0.2$; all $\left.\mathrm{p}<0.0005\right)$. Regarding changes in the

111 pRF size, a comparison across condition and visual areas revealed that the pRF size does not

112 change significantly between conditions $(\mathrm{F}(1,35)=0.007, \mathrm{p}=0.93)$ but it does change with visual

113 area $(\mathrm{F}(5,35)=6.5, \mathrm{p}<0.0001)$, and the interaction between condition and visual area is not 
114 significant $(\mathrm{F}(5,35)=0.63, \mathrm{p}=0.67)$. Post hoc tests (FDR corrected) did not show any significant

115 differences in $\mathrm{pRF}$ size between all the conditions tested $\mathrm{p}>0.09$ ).
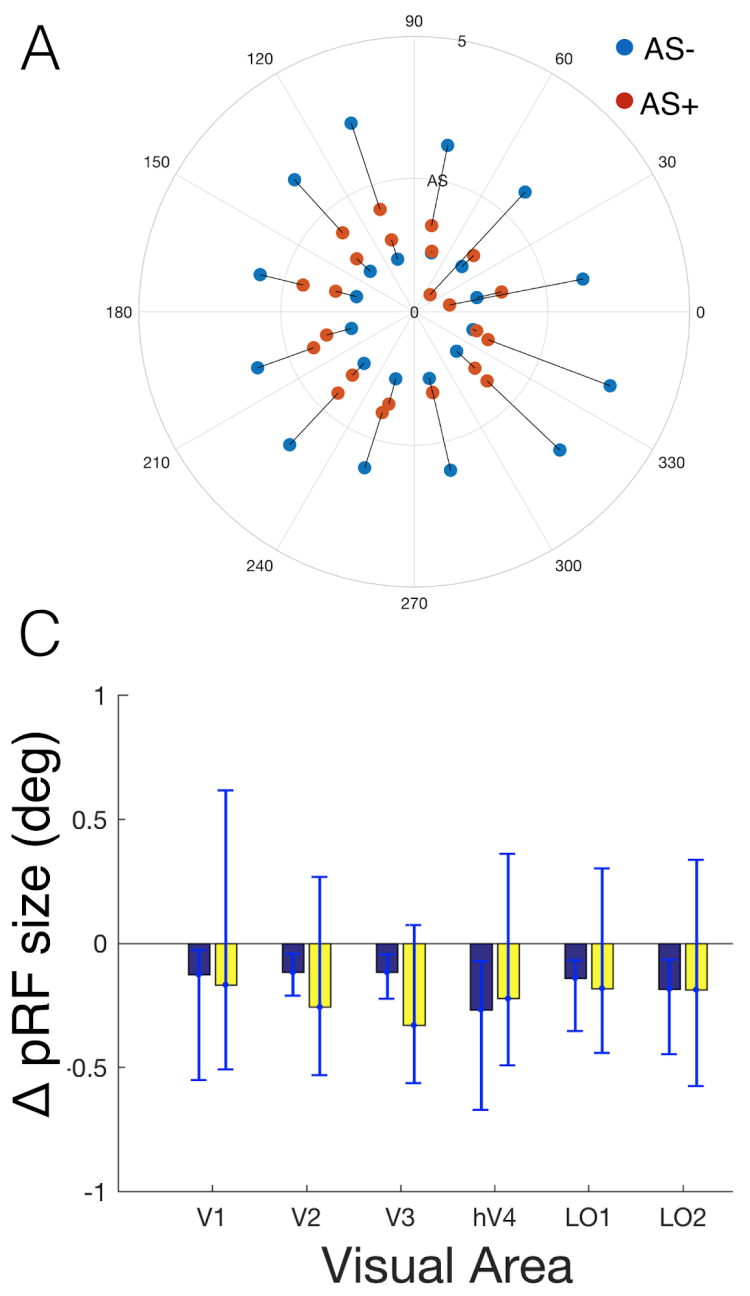
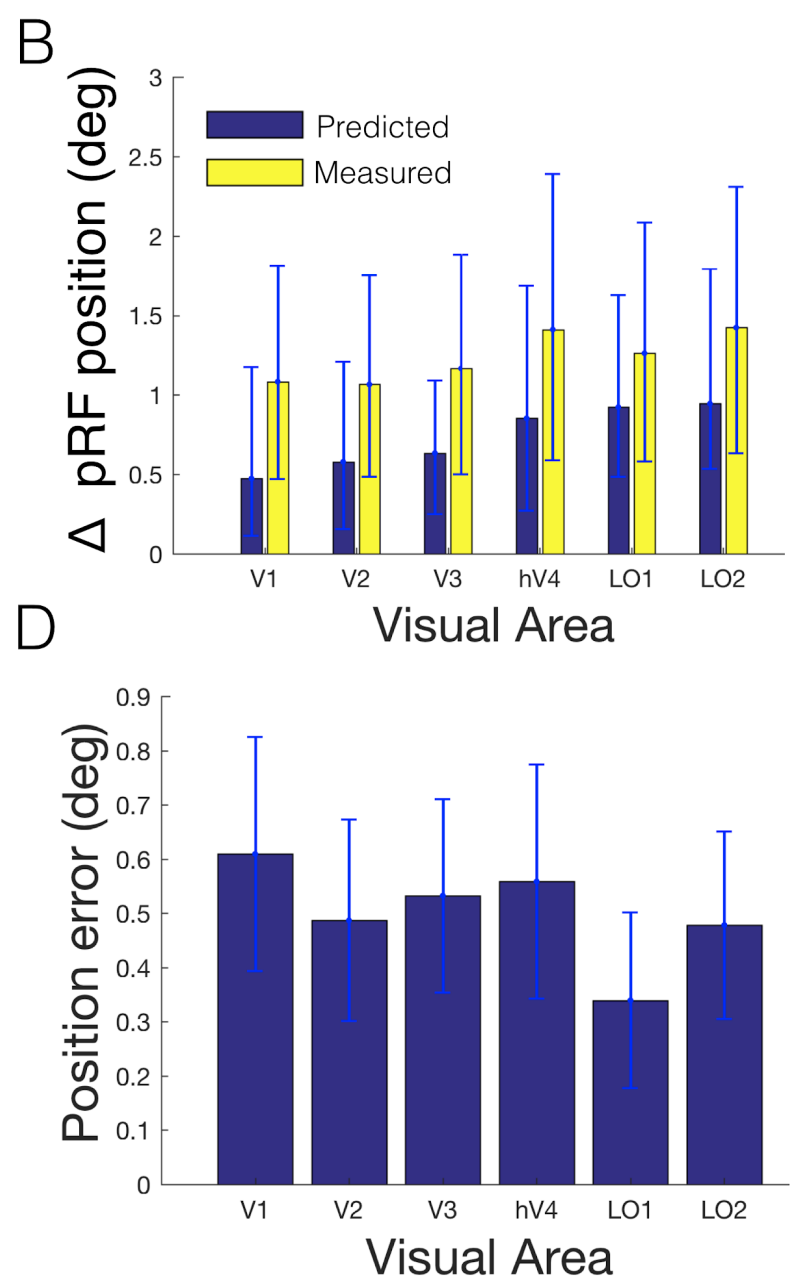

118 position change between the two conditions $\mathrm{AS}^{-}$(blue) and $\mathrm{AS}^{+}$(red) in various sectors of the visual field inside and outside the

119 AS, averaged across participants. The region inside the AS corresponds to the ASPZ. B/C: Measured (yellow) and predicted pRF 120 position shifts (B) and size changes (C) in response to an AS. D: Mean average error between the predicted and measured pRF

121 shifts. The error bars represent the interquartile ranges across the voxels in the test set. The estimated GF size did not vary 122 significantly between visual areas $(\mathrm{F}=0.16 ; \mathrm{p}=0.97))$. 


\section{Neural populations in extrastriate cortex increase their V1 sampling region}

124 Visual areas beyond V1 may also respond to the AS by changing their V1 sampling. Changes in 125 sampling of a source area such as V1 can be quantified by modeling the connective field (CF) of

126 the recording site. The CF enables the prediction of the neuronal activity of a recording site

127 (voxel) in a target region (e.g. V2) given the activity in another part of the brain (e.g. V1). CFs

128 are estimated without modeling the stimulus, so they are not subject to modeling bias and may

129 reflect other components of brain function, such as feedback signals. Changes in the CFs may

130 thus arise independently from the V1 pRF changes reported above. Figure 3A shows the

131 difference in CF size between the two AS conditions $\left(\mathrm{AS}^{+}-\mathrm{AS}^{-}\right)$for the voxels whose PP was

132 initially located either inside or outside the ASPZ. For some visual areas (voxels initially inside

133 the ASPZ) the CF became larger following the introduction of the AS. In particular, LO1

134 recording sites inside the ASPZ sampled from a larger region of V1, which is evident from the

135 increased CF size. This effect was not clearly present for recording sites outside the ASPZ (LO1:

136 inside ASPZ, $\mathrm{p}=0.002$; outside ASPZ $\mathrm{p}=0.14$ ). To show how the accumulation of these changes

137 influences the sampling of V1, we projected the CFs back into visual field space by convolving

138 them with the V1 pRFs from which they sample. To isolate AS-induced changes in the CFs from

139 those in the pRFs of $\mathrm{V} 1$, the CFs of both the $\mathrm{AS}^{-}$and $\mathrm{AS}^{+}$conditions were back projected using

140 the same set of pRFs (those from the $\mathrm{AS}^{-}$condition). For areas V2 and LO1, Figure 3B shows the

$141 \mathrm{CF}$ sampling density in the conditions $\mathrm{AS}^{-}$and $\mathrm{AS}^{+}$and their difference $\left(\mathrm{AS}^{+}-\mathrm{AS}^{-}\right)$. Overall, V2

142 sampling density is reduced in the $\mathrm{AS}^{+}$compared to the $\mathrm{AS}^{-}$condition. This effect is most

143 pronounced for recording sites within the ASPZ. The $\triangle \mathrm{CF}$ image shows that the introduction of

144 the AS generally resulted in a denser sampling of V1 regions outside the ASPZ. This effect

145 seems particularly pronounced in LO1. 

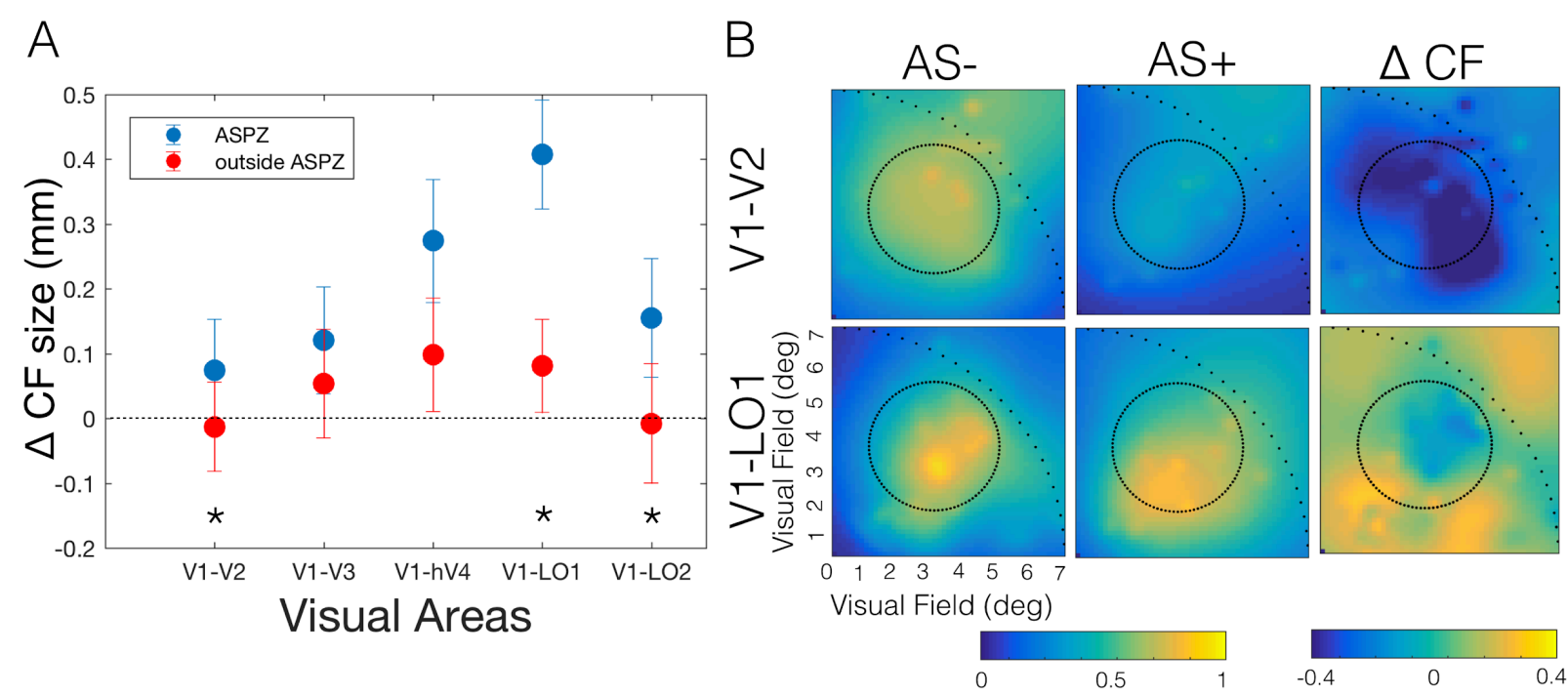

CF Sampling Density $\triangle$ CF Sampling Density

Figure 3. Changes in cortico-cortical connections in response to AS. A: Difference in the cumulative distribution at the $90 \%$ point (dashed line Figure S5A and B) of the CF size between $\mathrm{AS}^{+}$and $\mathrm{AS}^{-}$in (blue) and outside (red) the ASPZ. The error bars represent the $5 \%$ and $95 \% \mathrm{CI}$. The $\mathrm{CF}$ sizes between the two conditions $\left(\mathrm{AS}^{-}\right.$and $\left.\mathrm{AS}^{+}\right)$differed significantly inside and outside scotoma for V2, LO1 and LO2 $(\mathrm{p}<0.001)$, represented in the graph by *. Figures S5 A and B show the cumulative histogram of the CF sampling extent for ASPZ of the visual areas tested. Note that the V1 sampling extent increases (shift to the right) with visual hierarchy. This trend is not present for the voxels located outside the ASPZ (Figure S5B). The significance level between the two conditions $\left(\mathrm{AS}^{+}\right.$and $\mathrm{AS}^{-}$) per ROI is shown on the bottom right of the cumulative graphs. B: Coverage map of CFs obtained for $\mathrm{AS}^{-}, \mathrm{AS}^{+}$and the difference between the two conditions $\left(\mathrm{AS}^{+}-\mathrm{AS}^{-}\right)$. The back projection onto the visual field was performed based on the pRF estimates obtained with $\mathrm{AS}^{-}$. The sparse dotted line depicts the visual stimulation area and the dotted line the AS location in the visual field. Each map represents the combined data from 7 subjects.

\section{Discussion}

158 Our main finding is that in extrastriate cortical regions, in particular LO1, we observed increased 159 sampling of V1 regions outside the ASPZ, which would be required for the predictive masking 160 of the scotoma. Moreover, we find that inside and outside the ASPZ and throughout the visual 161 hierarchy, pRFs reconfigured their preferred spatial position and shifted it towards the AS 162 border. This behavior is inconsistent with what would be expected based on PM. However, a 
163 gain field model, centered at the AS border, could effectively explain these changes. This

164 suggests that the pRF changes primarily serve to focus neural resources on regions of potential

165 interest and constitute a component of normal visual perception. The model explained the shifts

166 most effectively for extrastriate areas, in particular for area LO1. We therefore postulate that the

167 population modifications originate in extrastriate areas and, through feedback, also modulate the

168 V1 pRFs. Therefore, changes in intra-area connectivity (connective fields), rather than those of

169 the pRFs, may be the neural underpinning of PM. In summary, our results reveal an extended,

170 system-wide reconfiguration of neural population properties in response to the change in visual

171 input evoked by an AS. Below, we discuss our findings and interpretation in detail.

\section{Extrastriate cortex increased its sampling of V1 outside of the ASPZ}

173 To understand how the cortico-cortical connections between visual areas change in response to

174 an AS, we quantified their CFs, which describe how extrastriate target areas (V2 to LO2) sample

175 from source area V1. Dissociating the changes in the extrastriate CFs from their pRF shifts

176 revealed an increased sampling density of the V1 region outside of the ASPZ in response to the

177 AS. This effect was particularly evident for LO1, where the sampling from V1 increased

178 especially for voxels inside the ASPZ. This indicates that cortico-cortical connections change

179 following the presentation of the AS, resulting in increased capturing of information from

180 outside the scotomatic region. This is consistent with PM.

181 The capacity to dissociate connectivity from the visual input via the back projection of the CFs is

182 less susceptible to stimulus-related model-fitting biases (due to its independence from the

183 stimulus) and informs how the visual information is integrated across different cortical areas. It 
184 also has the potential to capture the neural circuits underlying pRF dynamics (Carvalho et al., in 185 press).

\section{Feedback from extrastriate regions drives system-wide reconfiguration}

187 Previous studies have reported dissociation in the representation of superficial, middle and deep

188 layers of V1. In these studies, the superficial and deep layers represented the feedback

189 mechanisms that modulate perception, and the middle layers represented the visual input.

190 Evidence of predictive feedback in the superficial layers of V1 was found when neurons were

191 deprived of information in a partial occlusion paradigm $(26,27)$. Selective feedback-associated

192 activation of the deep layers of V1 was also found in a study on the Kanizsa illusion (28).

193 Therefore, the pRF changes measured in the early visual cortex could plausibly be driven by

194 feedback connections from extrastriate cortex. Moreover, based on our results and those of

195 others, extrastriate area LO1 is a potential candidate for the origin of these feedback signals. It

196 plays a major role in the processing of oriented boundaries or borders $(29,30)$ and its role can be

197 dissociated from that of LO2, which preferably processes shape (30). In our analysis, the gain

198 field model best explained the observed pRF modulations in this area, which would be expected

199 for signals originating in this area. Moreover, the increased sampling of V1 was most prominent

200 for LO1 voxels. We therefore propose that the reconfiguration of neural populations in response

201 to an AS is modulated by extrastriate signals and may underlie predictive masking.

202 Although PM is linked to perceptual filling-in (FI), we opted to not quantify perceptual FI during

203 our experiments. This is because such a perceptual task could interfere with the attention task

204 and increase the chance of unintentional small eye-movements in the direction the AS, thereby

205 actually decreasing FI. Therefore, we performed psychophysical tests outside the scanner and 
206 prior to the present study, which indicated that participants reach stable FI after about 30 sec

207 (Figure S6). Since our actual mapping experiment started after $60 \mathrm{sec}$ and the design of the

208 retinotopic stimulus was optimized to yield FI, we assumed that observers were filling in the AS

209 at the time we performed the pRF and CF mapping.

210 An artificial scotoma induces a system-wide reconfiguration of neural population

211 receptive fields

212 In response to the AS, pRFs shift their preferred spatial position towards the AS border. While

213 such shifts have been reported previously for pRFs initially located inside the natural SPZ (31)

214 and ASPZ $(10-12,32)$, our study is the first to show that this reconfiguration is not restricted to

215 the ASPZ, but is a system-wide phenomenon. Within the ASPZ, the pRFs shifted their preferred

216 position towards the AS border, which could be consistent with an extrapolation process.

217 Following the shift, pRFs are more likely to be activated by spared portions of the visual field,

218 and can thus contribute to the spatial masking of the scotoma. However, the pRFs initially

219 located outside the ASPZ shifted their preferred position towards the AS border as well. These

220 pRFs are more likely activated by non-stimulated portions of the visual field. Therefore, this

221 behavior cannot easily be reconciled with PM.

222 Previous studies have suggested that changes in the pRF properties in response to an AS can

223 result from a model bias driven by partial stimulation of the neuronal populations $(11,12,32)$.

224 This effect can be controlled by taking into account the presence of the AS during the pRF

225 modeling $(12,32)$. Accordingly, we used two pRF modeling approaches: one that assumed the

226 presence of the AS - the Scotoma Field (SF) model, and one that did not - the Full Field (FF)

227 model. We found similar positional shifts with both models, thus indicating that our findings are

228 unbiased (Figure S4). Importantly, CFs are not affected by such model biases. 


\section{A gain field at the scotoma border explains the shifts in $p R F$ preferred position}

230 The factor common to all shifts is that these were predominantly directed towards the AS border.

231 Indeed, the PP changes could be explained by a biologically motivated GF that accounts for the

232 presence of the AS. This suggests that the presence of an AS results in a reweighting of the

233 spatial response selectivity towards the scotomatic border. Similar results were found using a

234 model of attention $(25,33)$. Therefore, the presence of the AS could result in a deployment of

235 attention towards the AS border. Although the AS was designed to induce PM (filling-in), a

236 reduced visual stimulation may actually be salient to the early visual system (34). In this case,

237 the PP shifts indicate that the border was a salient feature. This interpretation is supported by the

238 fact that GF model described the PP shifts accurately, especially for the extrastriate areas. This

239 interpretation is also in line with previous studies, which showed that high-level mechanisms

240 (attention) modulate perception via feedback projections (20). The reconfiguration of neural

241 population properties may therefore have the more general role of allocating neural resources to

242 salient features in the visual field. This may help to scrutinize these in more detail, or

243 alternatively, to resolve prediction errors (35).

244 This interpretation links to previous hypotheses about the underlying mechanisms of PM, in

245 particular the suggestion that the masking of an AS results from (slow - tenths of seconds)

246 adaptation to salient features (such as a border) in combination with a fast extrapolation process

247 (36). Although, the design of the present experiment did not allow us to separate these two

248 components, the GF model can shed some light on these issues. We suggest that during the

249 border adaptation, neural resources are allocated to the borders of the scotoma in response to its

250 saliency, resulting in a reconfiguration of the RFs and consequently in the predictive spatial

251 masking of the scotoma. These findings indicate that the modulation of the pRF structure by 
252 cognitive factors contributes to the adaptation to the scotoma borders and consequently to the

253 predictive masking.

254 In contrast to previous studies using retinal and cortical scotomas $(5,8)$, our observed PP shifts

255 were not accompanied by increases in pRF size (if something they tended to shrink). The

256 absence of size changes in early visual cortex may be related to our use of a low spatial

257 frequency stimulus. Therefore, the most responsive neurons defining the pRF already had large

258 receptive fields, leaving little room for further expansion. Importantly, the presence of the AS

259 did not alter fundamental structural characteristics of the visual cortex, such as the increase of the

260 pRF size over eccentricity and visual hierarchy. However this last aspect does not explain the

261 increase of the position shifts over the visual hierarchy.

\section{Limitations and future studies}

263 Eye movements may bias pRF estimates and commonly result in increased pRF sizes $(25,38$,

264 39). Eye movements were not recorded during scanning but were minimized by having observers

265 perform an attention task that demanded central fixation. Moreover, eye movement artifacts

266 should have resulted in increased pRF sizes, which we did not find.

267 For five of the seven observers the $\mathrm{AS}^{-}$and $\mathrm{AS}^{+}$conditions were performed in two different scan

268 sessions raising the possibility that pRF shifts were due to misalignment between the functional

269 and anatomical scans. However, such shifts should all have been in the same direction, e.g. fovea

270 to periphery. Moreover, we find similar shifts in the two observers who performed the two

271 conditions within the same scan session. Therefore, we conclude that the observed pRF shifts are

272 genuine. 
273 We defined the pRFs contained by the ASPZ based on the pRF estimates obtained with the AS $^{-}$

274 condition. As an alternative method, we also defined the ASPZ based on a scotoma localizer in

275 which the AS and its background were stimulated separately. The results obtained using either

276 definition of the ASPZ resulted in highly analogous findings, reason why we choose to present

277 the results based on only one method.

278 Future studies measuring the neuronal mechanisms associated with PM at finer scale (e.g. at

279 higher fMRI resolution) could reveal changes that are masked at a coarser scale. This is not only

280 because one can identify more pRFs in the ASPZ, but also because it enables determining

281 laminar profiles across cortical depth, which could help to determine at which level of cortical

282 processing the feedback and feedforward signals modulate perception.

283 In conclusion, in the present study we have shown that partial occlusion of local visual input

284 results in a system-wide reconfiguration of the RF properties of neural populations and their

285 connectivity. Furthermore, we suggest that this reconfiguration is guided by extrastriate signals,

286 that the reconfiguation is an integral component of normal perception and that it forms the basis

287 of predictive masking in health and disease.

288 Materials and Methods

290 Seven participants (3 females; average age: 28; age-range: 26-32) with normal or corrected-to-

291 normal vision were included in the study. The participants indicated that they understood the

292 instructions. Prior to participation, participants signed an informed consent form. Our study was 
approved by the Medical Ethical Review Board of the University Medical Center of Groningen,

294 and conducted in accordance with the Declaration of Helsinki.

295

296

297

298

299

300

301

302

303

304

305

306

307

308

309

310

\section{1}

312

313

\section{Data acquisition}

Stimuli were presented on an MR compatible display screen (BOLDscreen 24 LCD; Cambridge Research Systems, Cambridge, UK). The screen was located at the head-end of the MRI scanner. Participants viewed the screen through a tilted mirror attached to the head coil. Distance from the participant's eyes to the display (measured through the mirror) was $120 \mathrm{~cm}$. Screen size was $22 \times 14 \mathrm{deg}$. The maximum stimulus radius was $7 \mathrm{deg}$ of visual angle. Visual stimuli were created using MATLAB (Mathworks, Natick, MA, USA) and the Psychtoolbox (40, 41).

\section{Stimuli}

\section{Luminance-contrast defined retinotopy (LCR)}

LCR consists of a drifting bar aperture defined by high-contrast flickering texture (42). The bar aperture, i.e. alternating rows of high-contrast luminance checks drifting in opposite directions, moved in 8 different directions (four bar orientations: horizontal, vertical and the two diagonal orientations), with two opposite drift directions for each orientation (Figure 4A). The bar moved across the screen in 16 equally spaced steps each lasting 1 TR. The bar contrast, width and spatial frequency were $100 \%, 1.75$ and 0.5 cycles per degree, respectively. After 24 steps (one pass and a half), $12 \mathrm{~s}$ of a blank full screen stimulus at mean luminance was presented.

\section{Artificial Scotoma (AS) conditions}

The stimuli used in the two AS conditions were adapted from the LCR stimulus. More specifically, the bar and background could be distinguished from each other only on the basis of 
314 their spatial frequency (Figure 4B). The $\mathrm{AS}^{-}$condition served as the control condition for the

$315 \mathrm{AS}^{+}$condition that contained the actual scotoma. The bar's movement directions and orientations

316 matched those of the LCR condition. The width of the bar aperture was 3 degrees. The bar

317 content was dynamic white-noise band passed filtered at frequencies from 0 to 2 cycles per

318 degree (cpd). The background consisted of dynamic white SF band passed from 2 to 4 cpd. The

319 long edges of the bar were smoothed using an exponential mask. The formula for this mask was:

$320=e^{-\frac{r f}{2}}$, where $\mathrm{r}$ is the distance to the center-line of the bar, and $f$ the mask factor. The value of

$321 f$ was fixed at 4 . The bar moved at a speed of $0.46 \mathrm{deg} / \mathrm{sec}$. The AS- condition was used to

322 define a baseline PP and size of the pRF for each voxel. The $\mathrm{AS}^{+}$condition was similar to $\mathrm{AS}^{-}$

323 (with equal bar aperture size, movement and SF). Four ASs were superimposed on the dynamic

324 noise background (see Figure 4C). The scotomas were centered at each quarter field at 4.5 deg of

325 eccentricity. Each AS consisted of 2.5 deg radius disc tapered by an exponential mask at the

326 edges, similar to the masking of the bar: $=e^{-\frac{r^{f}}{2}}$, where, $\mathrm{r}$ is the distance from the center of the

327 scotoma and $\mathrm{f}$ is fixed at a value of four, as before. Preceding each run was a one-minute

328 adaptation period during which the participants viewed only the background with the AS

329 superimposed while performing the fixation attentional task. In psychophysical experiments,

330 performed prior to the fMRI scans, we determined that this period was sufficient to induce

331 filling-in (see Figure S6).

\section{Attentional task}

333 During scanning, participants were required to perform a fixation task in which they had to press

334 a button each time the fixation point turned from green to red. The average performance on this 

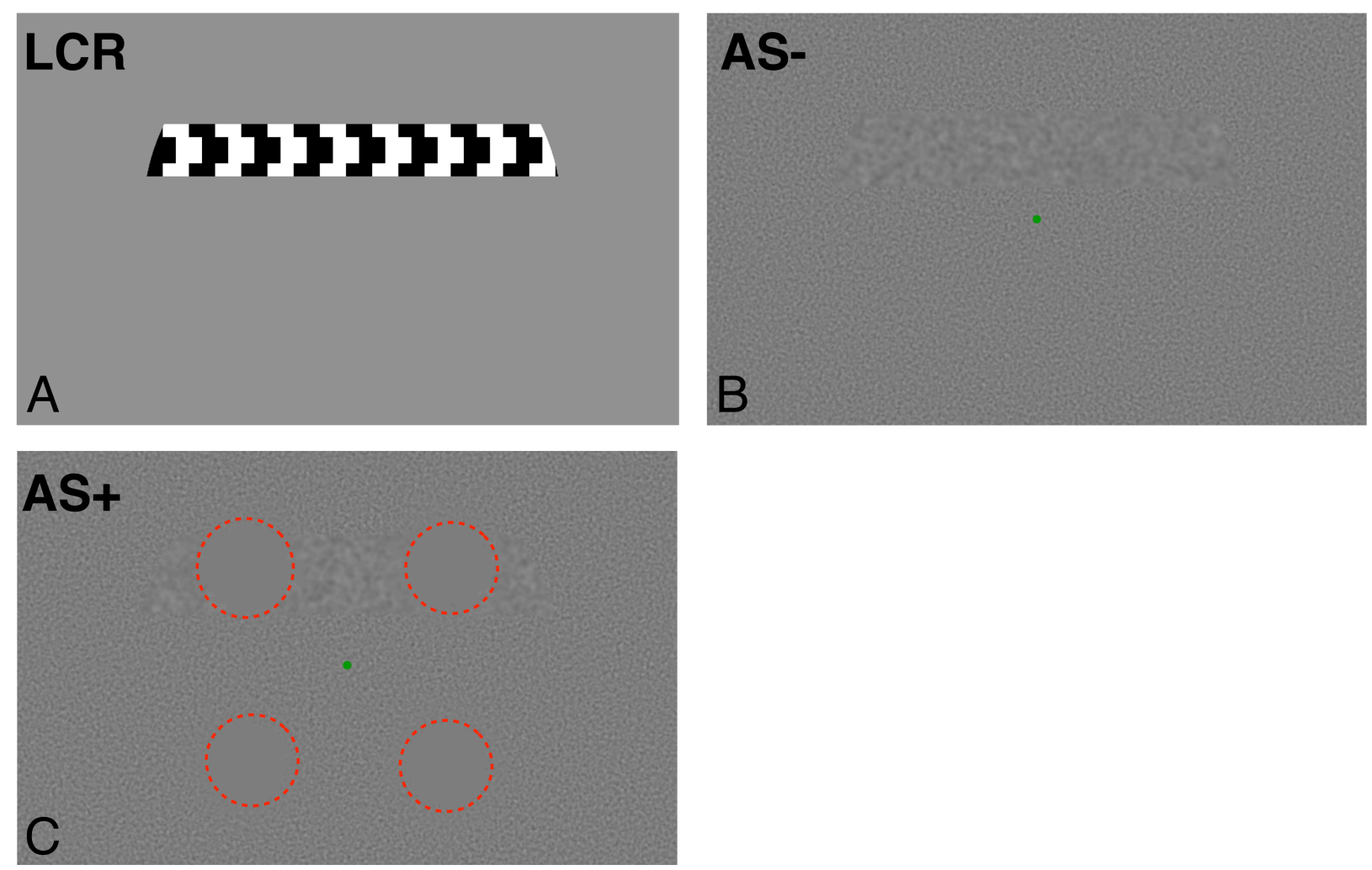

Figure 4. Example of the stimuli used to obtain pRF parameter estimates. A: LCR; B: $\mathrm{AS}^{-}$, $\mathrm{C}^{\text {: }} \mathrm{AS}^{+}$, for visualization purposes the AS are outlined with a dashed red line. NB: this red dashed line was not presented to the participants

$340 \quad$ MRI scanning and preprocessing

341 Scanning was carried out on a 3 Tesla Siemens Prisma MR-scanner using a 64-channel receiving

342 head coil. A T1-weighted scan (voxel size, $1 \mathrm{~mm}^{3}$; matrix size, $256 \times 256$ x 256) covering the

343 whole brain was recorded to chart each participant's cortical anatomy. The functional scans were

344 collected using standard EPI sequence (TR, $1500 \mathrm{~ms}$; TE, $30 \mathrm{~ms}$; voxel size, $3 \mathrm{~mm}^{3}$, flip angle

34580 ; matrix size, $84 \times 84 \times 24$ ). Slices were oriented to be approximately parallel to the calcarine

346 sulcus. For the retinotopic scans LCR and $\mathrm{AS}^{-}$a single run consisted of 136 functional images

347 (duration of $204 \mathrm{~s}$ ) and for $\mathrm{AS}^{+}$a single run consisted on 168 functional images (252 s). 
348 The T1-weighted whole-brain anatomical images were re-sampled to a $1 \mathrm{~mm}^{3}$ resolution. The

349 resulting anatomical image was automatically segmented using Freesurfer (43) and subsequently

350 edited manually. The cortical surface was reconstructed at the gray/white matter boundary and

351 rendered as a smoothed 3D mesh (44).

352 The functional scans were analyzed in the mrVista software package for MATLAB (available at 353 http://white. stanford.edu/software). Head movements between and within functional scans were

354 corrected (45). The functional scans were averaged and co-registered to the anatomical scan (45),

355 and interpolated to a $1 \mathrm{~mm}$ isotropic resolution. Drift correction was performed by detrending the

356 BOLD time series with a discrete cosine transform filter with a cutoff frequency of $0.001 \mathrm{~Hz}$. To

357 avoid possible saturation effects, initial images were discarded for the LCR and $\mathrm{AS}^{-}$(8 $\mathrm{TRs}^{\text {), as }}$

358 well as for the $\mathrm{AS}^{+}$(40 TRs). Note that the full 60 seconds adaptation period was removed for 359 the $\mathrm{AS}^{+}$.

\section{Experimental procedure}

361 Each participant completed two fMRI sessions of approximately $1.5 \mathrm{~h}$. In the first fMRI session,

3625 participants were subjected to the anatomical scan and LCR, and they performed the $\mathrm{AS}^{-}$ 363 experiment (6 runs, $3.4 \mathrm{~min}$ each). In the second fMRI session, the $\mathrm{AS}^{+}$experiment (6 runs, 4.2

364 min each) were performed. To eliminate the possibility that differences between conditions (AS

$365+$ and AS-) would result from the acquisition in different sessions, these were performed for 2

366 participants (S06 and S07) in the same session.

\section{Visual field mapping: $p R F$ modeling}

368 The pRF analysis was performed using both conventional pRF mapping (42) and a custom

369 implementation of the Monte Carlo Markov Chain (MCMC) Bayesian pRF approach (46, 47). In 
370 the conventional method, a 2D-gaussian model was fitted with parameters: center (x0, y0) and

371 size $(\sigma-$ width of the Gaussian) for each voxel. All the parameter units are in degrees of visual

372 angle and are stimulus-referred. We used SPM's canonical Haemodynamic Response Function

373 (HRF) model. The conventional pRF estimation was performed using the mrVista

374 (VISTASOFT) Matlab toolbox. The Bayesian pRF approach enables the estimation of the

375 uncertainty associated with each pRF parameter. The uncertainty was defined by the $25 \%$ and

$37675 \%$ quantiles of the estimated distribution.

377 In both approaches, the data was thresholded by retaining the pRF models that explained at least

$37815 \%$ of the variance. Furthermore, the functional responses to $\mathrm{LCR}, \mathrm{AS}^{-}$and $\mathrm{AS}^{+}$were analyzed

379 using the FF model. The $\mathrm{AS}^{+}$condition was also analyzed using the SF model (Figure 5A).

ROI and Artificial Scotoma Projection Zones definition

381 The cortical borders of visual areas were derived based on phase reversal, obtained with the

382 conventional pRF model using the classical the LCR stimulus. Per observer, six visual areas (V1,

$383 \mathrm{~V} 2, \mathrm{~V} 3, \mathrm{~V} 4, \mathrm{LO} 1$ and LO2) were manually delineated on the inflated cortical surface.

384 Based on the pRF estimates obtained with the AS- condition, the ASPZ was defined as the voxels

385 for which the pRF was completely contained within the AS regions of the visual field.

\section{Gain Field model}

387 The influence of the AS on the pRF's preferred position and size was modeled as a gain field 388 (GF), i.e., the multiplication of two Gaussian components $(25,33,37,48)$. In our study, the first

389 Gaussian component corresponded to the $\mathrm{pRF}$ estimated in the $\mathrm{AS}^{-}$condition $\left(u_{A S_{-},}, \sigma_{A S_{-}}\right)$. The 390 second Gaussian component corresponded to the GF $\left(u_{G F}, \sigma_{G F}\right)$ elicited by the AS: it represented 
391 the influence of the AS on the pRF's preferred position. The GF was centered on the border of

392 the AS at the point nearest to the original pRF location (Figure 5). The product of these two

393 components resulted in a third Gaussian $\left(u_{p A S+}, \sigma_{p A S+}\right)$, that represented the predicted pRF in

394 the $\mathrm{AS}^{+}$condition. Equations 1 and 2 show how the properties of the third Gaussian were

395 derived.

$$
u_{p A S+}=\left(\frac{u_{A S-} * \sigma_{G F}^{2}+u_{G F} * \sigma_{A S-}^{2}}{\sigma_{G F}^{2}+\sigma_{A S-}^{2}}\right)
$$

$$
\sigma_{p A S+}=\sqrt{\left(\frac{\sigma_{G F}^{2} * \sigma_{A S-}^{2}}{\sigma_{G F}^{2}+\sigma_{A S-}^{2}}\right)}
$$

398 The GF size was estimated by minimizing the error between the predicted and the measured 399 position shifts, which is the radial distance between the $\mathrm{AS}^{+}$and $\mathrm{AS}^{-}$. For verification of the 400 model's accuracy, the data was split into a training set (50\% of the data) and a test set (the 401 remaining $50 \%$ of the data).
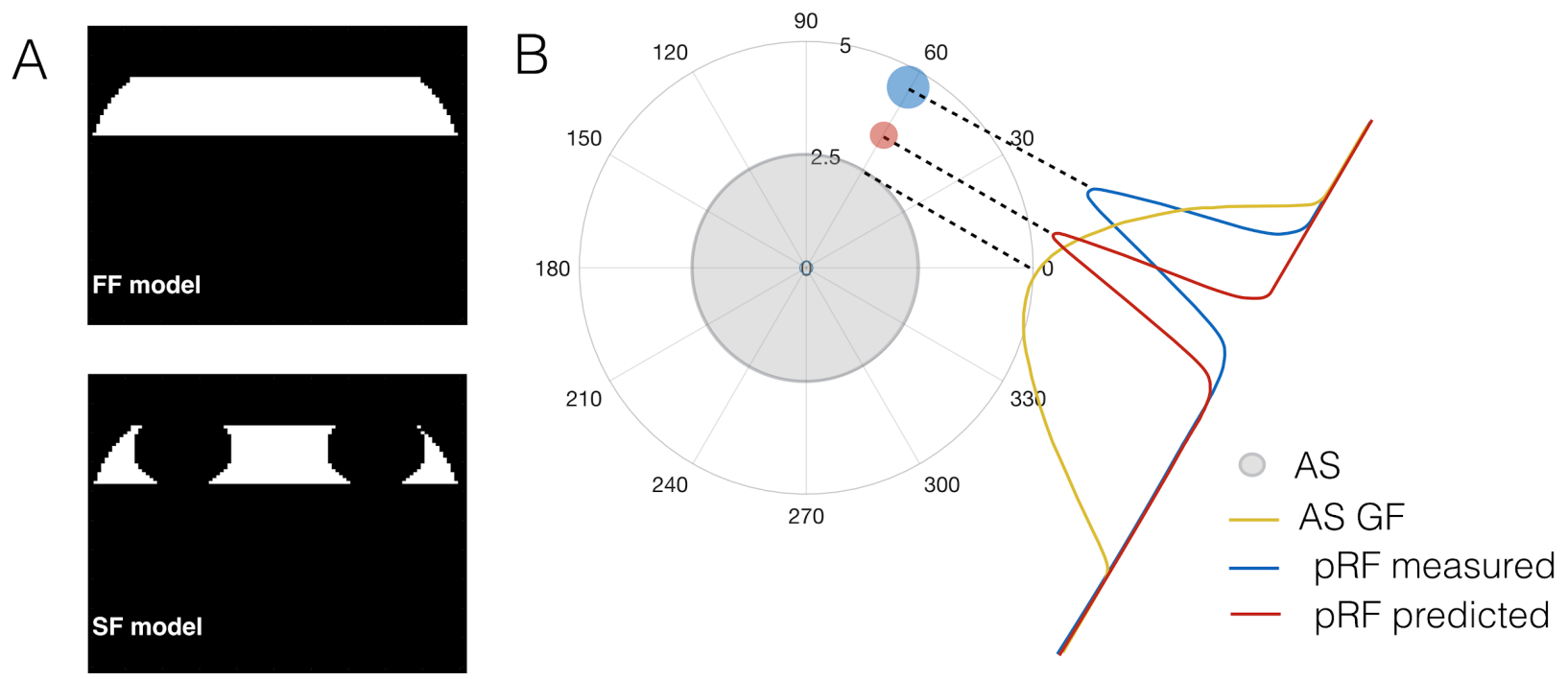

Figure 2. Models of neural responses used in the analysis, FF, SF and AS Gain Field model. A: The full field (FF) and scotoma field (SF) models used in the pRF analysis. B: AS GF model: the AS (shaded grey region) effect was modeled as the AS GF (yellow), centered at the edge of the scotoma closest to the pRF (blue). This results in a predicted pRF (red), shifted towards 
408 The CF model predicts the neuronal activity of a recording site (voxel) in a target region (e.g. in

409 V2) given the aggregate activity in a source region (in V1) (49). The fMRI response of each

410 voxel is predicted using a 2D circular Gaussian CF model, folded to follow the cortical surface

411 of the source region. The CF output parameters are the position and spread (size) across the

412 source surface. Given a CF position and a size, a time-series prediction is then calculated by

413 weighting the $\mathrm{CF}$ with the BOLD time series. The optimal CF parameters are found by

414 minimizing the residual sum of squares between the predicted and the measured time-series. In

415 this study, only CFs with a VE $>0.6$ were retained.

\section{Statistical analysis}

417 Data was thresholded by retaining the pRF models that explained at least $15 \%$ of the variance in

418 the BOLD response in the three conditions ( $\left.\mathrm{LCR}, \mathrm{AS}^{+}, \mathrm{AS}^{-}\right)$. For the analysis of changes in $\mathrm{pRF}$

419 properties in response to the AS, the $\mathrm{pRF}$ estimates of the four quadrants were collapsed onto a

420 single quadrant. Subsequently, voxels were binned into 12 bins, each covering an eccentricity

421 range of $1.75 \mathrm{deg}$ and a polar angle range of $30^{\circ}$ (Figure 3B). Additionally within the ASPZ,

422 voxels were binned into 12 bins of $30 \mathrm{deg}$ of polar angle each after shifting the origin to the

423 center of the ASPZ (Figure 3A).

424 The PP change corresponds to the Euclidean or radial distance between the $\mathrm{AS}^{+}$and $\mathrm{AS}^{-}$

425 conditions. The size ratio, $\sigma_{r}$, was calculated based on the following equation:

$$
\sigma_{r}=\left(\frac{\sigma_{A S_{+}}-\sigma_{A S-}}{\sigma_{A S-}}\right)
$$


427 The CF coverage maps were obtained by back projecting each CF into the visual space using the

428 pRFs for V1 obtained with $\mathrm{AS}^{-}$. First, per voxel in the target region, a CF was calculated, i.e. the

429 target voxel is expressed as the weighted (CF factor) average of the signals measured in V1 (the

430 source region). As the $\mathrm{pRF}$ was known for each voxel in V1, we calculated the spatial sampling

431 by summing all pRFs of V1 weighted by the CF factor. The total CF coverage map was

432 calculated by summing these maps across all voxels in the target region. Finally, a group average

$433(\mathrm{n}=7)$ was calculated across subjects.

434 Repeated measures ANOVA, with ROI, condition ( $\left.\mathrm{AS}^{-}, \mathrm{AS}^{+} \mathrm{SF}\right)$, hemisphere and position bin as

435 within-subject parameters, was used to compare the difference of the pRF preferred position and

436 size between conditions. Subjects were treated as random variables. For the $\mathrm{AS}^{+}$condition, the

437 pRF properties were estimated using two different models (FF, SF Figure 2A). Separate

438 statistical analyses were performed for each of the resulting parameter sets. Permutation tests

439 (1000 replications) were used to determine significance level of the differences in CF size

440 between conditions inside and outside the ASPZ. For this, data was aggregated over participants

441 and condition labels were permuted.

442 All analyses were performed using MATLAB (version 2016b; Mathworks, Natick, MA, USA)

443 and R (version 2.11.1; R Foundation for Statistical Computing, Vienna, Austria). A p-value of 0.05

444 or less was considered significant.

\section{Acknowledgments}

446 Author JC was supported by the European Union's Horizon 2020 research and innovation

447 programme under the Marie Sklodowska-Curie grant agreement No. 641805 (NextGenVis) 
awarded to FWC. The funding organization had no role in the design, conduct, analysis, or

449 publication of this research.

\section{References}

451

1. Hoste AM (2003) New insights into the subjective perception of visual field defects. Bull Soc Belge Ophtalmol (287):65-71.

2. Smith ND, Crabb DP, Glen FC, Burton R, Garway-Heath DF (2012) Eye movements in patients with glaucoma when viewing images of everyday scenes. Seeing Perceiving 25(5):471-492.

3. Pessoa L, De Weerd P (2003) Filling-In: From Perceptual Completion to Cortical Reorganization (Oxford University Press).

4. Komatsu H (2006) The neural mechanisms of perceptual filling-in. Nat Rev Neurosci 7(3):220-231.

5. Gilbert CD, Wiesel TN (1992) Receptive field dynamics in adult primary visual cortex. Nature 356(6365):150-152.

6. Schumacher EH, et al. (2008) Reorganization of visual processing is related to eccentric viewing in patients with macular degeneration. Restor Neurol Neurosci 26(4-5):391-402.

7. Dilks DD, Baker CI, Peli E, Kanwisher N (2009) Reorganization of Visual Processing in Macular Degeneration Is Not Specific to the "Preferred Retinal Locus." J Neurosci 29(9):2768-2773.

8. Pettet MW, Gilbert CD (1992) Dynamic changes in receptive-field size in cat primary visual cortex. Proc Natl Acad Sci U S A 89(17):8366-8370.

9. Baker CI, Peli E, Knouf N, Kanwisher NG (2005) Reorganization of visual processing in macular degeneration. J Neurosci 25(3):614-618.

10. Baseler HA, et al. (2011) Large-scale remapping of visual cortex is absent in adult humans with macular degeneration. Nat Neurosci 14(5):649-655.

11. Haak KV, Cornelissen FW, Morland AB (2012) Population receptive field dynamics in human visual cortex. PLoS One 7(5):e37686.

12. Papanikolaou A, Keliris GA, Lee S, Logothetis NK, Smirnakis SM (2015) Nonlinear population receptive field changes in human area $\mathrm{V} 5 / \mathrm{MT}+$ of healthy subjects with simulated visual field scotomas. Neuroimage 120:176-190.

13. Das A, Gilbert CD (1995) Long-range horizontal connections and their role in cortical reorganization revealed by optical recording of cat primary visual cortex. Nature 375(6534):780-784.

14. Tremere LA, Pinaud R, De Weerd P (2003) Contributions of inhibitory mechanisms to perceptual completion and cortical reorganization. Filling-in: from perceptual completion to cortical reorganization (Pessoa L, De Weerd P, eds):295-322. 
15. Weil RS, Watkins S, Rees G (2008) Neural correlates of perceptual completion of an artificial scotoma in human visual cortex measured using functional MRI. Neuroimage 42(4):1519-1528.

16. Parks NA, Corballis PM (2012) Neural Mechanisms of Short-term Plasticity in the Human Visual

17. Smirnakis SM, et al. (2005) Lack of long-term cortical reorganization after macaque retinal lesions. Nature 435(7040):300-307.

18. Mendola JD, Conner IP, Sharma S, Bahekar A, Lemieux S (2006) fMRI Measures of perceptual filling-in in the human visual cortex. J Cogn Neurosci 18(3):363-375.

19. Weil RS, Kilner JM, Haynes JD, Rees G (2007) Neural correlates of perceptual filling-in of an artificial scotoma in humans. Proc Natl Acad Sci U S A 104(12):5211-5216.

20. De Weerd P, Smith E, Greenberg P (2006) Effects of selective attention on perceptual filling-in. $J$ Cogn Neurosci 18(3):335-347.

21. Harrison WJ, Ayeni AJ, Bex PJ (2019) Attentional selection and illusory surface appearance. Sci $\operatorname{Rep} 9(1): 2227$.

22. Wandell BA, Smirnakis SM (2009) Plasticity and stability of visual field maps in adult primary visual cortex. Nat Rev Neurosci 10(12):873-884.

23. Chen Y, et al. (2009) The linearity and selectivity of neuronal responses in awake visual cortex. J Vis 9(9):12.1-17.

24. Enroth-Cugell C, Freeman AW (1987) The receptive-field spatial structure of cat retinal Y cells. The Journal of Physiology 384(1):49-79.

25. Klein BP, Harvey BM, Dumoulin SO (2014) Attraction of position preference by spatial attention throughout human visual cortex. Neuron 84(1):227-237.

26. Muckli L, et al. (2015) Contextual Feedback to Superficial Layers of V1. Curr Biol 25(20):26902695.

27. Morgan A, Petro L, Muckli L (2018) Cortical feedback to superficial layers of V1 contains predictive scene information. 2018 Conference on Cognitive Computational Neuroscience. doi:10.32470/ccn.2018.1083-0.

28. Kok P, Bains LJ, van Mourik T, Norris DG, de Lange FP (2016) Selective Activation of the Deep

29. Larsson J, Heeger DJ (2006) Two retinotopic visual areas in human lateral occipital cortex. $J$ Layers of the Human Primary Visual Cortex by Top-Down Feedback. Curr Biol 26(3):371-376. Neurosci 26(51):13128-13142.

30. Silson EH, et al. (2013) Specialized and independent processing of orientation and shape in visual field maps LO1 and LO2. Nat Neurosci 16(3):267-269.

31. Barton B, Brewer AA (2015) fMRI of the rod scotoma elucidates cortical rod pathways and implications for lesion measurements. Proc Natl Acad Sci U S A 112(16):5201-5206.

515 32. Binda P, Thomas JM, Boynton GM, Fine I (2013) Minimizing biases in estimating the 
reorganization of human visual areas with BOLD retinotopic mapping. $J$ Vis 13(7):13.

33. Womelsdorf T, Anton-Erxleben K, Pieper F, Treue S (2006) Dynamic shifts of visual receptive fields in cortical area MT by spatial attention. Nature Neuroscience 9(9):1156-1160.

34. Betz T, Wilming N, Bogler C, Haynes J-D, König P (2013) Dissociation between saliency signals and activity in early visual cortex. $J$ Vis.

35. Haak KV, Morland AB, Rubin GS, Cornelissen FW (2016) Preserved retinotopic brain connectivity

36. Spillmann L, De Weerd P (2003) Mechanisms of Surface Completion: Perceptual Filling-In of Texture. Filling-In:81-105.

37. Womelsdorf T, Anton-Erxleben K, Treue S (2008) Receptive field shift and shrinkage in macaque middle temporal area through attentional gain modulation. $J$ Neurosci 28(36):8934-8944.

38. Hummer A, et al. (2016) Eyetracker-based gaze correction for robust mapping of population receptive fields. Neuroimage 142:211-224.

39. Levin N, Dumoulin SO, Winawer J, Dougherty RF, Wandell BA (2010) Cortical maps and white matter tracts following long period of visual deprivation and retinal image restoration. Neuron 65(1):21-31.

40. Brainard DH (1997) The Psychophysics Toolbox. Spat Vis 10(4):433-436.

41. Pelli DG (1997) The VideoToolbox software for visual psychophysics: transforming numbers into movies. Spat Vis 10(4):437-442.

42. Dumoulin SO, Wandell BA (2008) Population receptive field estimates in human visual cortex. Neuroimage 39(2):647-660.

43. Dale AM, Fischl B, Sereno MI (1999) Cortical surface-based analysis. I. Segmentation and surface reconstruction. Neuroimage 9(2):179-194.

44. Wandell BA, Chial S, Backus BT (2000) Visualization and measurement of the cortical surface. $J$

45. Nestares O, Heeger DJ (2000) Robust multiresolution alignment of MRI brain volumes. Magn Reson Med 43(5):705-715.

46. Zeidman P, Silson EH, Schwarzkopf DS, Baker CI, Penny W (2018) Bayesian population receptive

47. Adaszewski S, Slater D, Melie-Garcia L, Draganski B, Bogorodzki P (2018) Simultaneous estimation of population receptive field and hemodynamic parameters from single point BOLD responses using Metropolis-Hastings sampling. Neuroimage 172:175-193.

548 48. Reynolds JH, Heeger DJ (2009) The normalization model of attention. Neuron 61(2):168-185.

549 49. Haak KV, et al. (2013) Connective field modeling. Neuroimage 66:376-384. 


\section{Supplementary material}

551 Contents

552 1. Shifts in PP of pRFs occur throughout the visual hierarchy

553 2. Simulations of pRF shifts

554 3. Comparison between SF and FF model analyses

555 4. Connective fields in extrastriate cortex increase their sampling extent.

556 5. Filling-in time

557 6. Attention task performance 


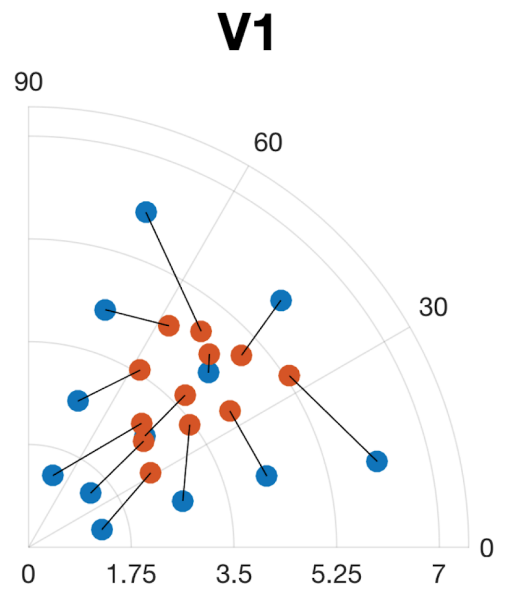

V4

90

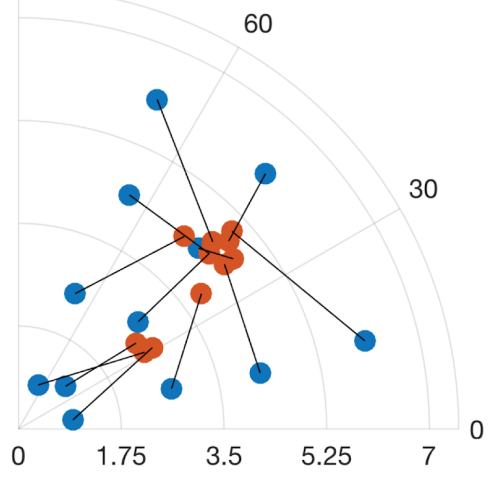

90

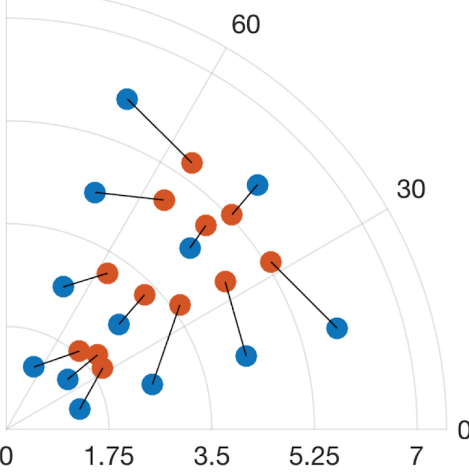

L01

90

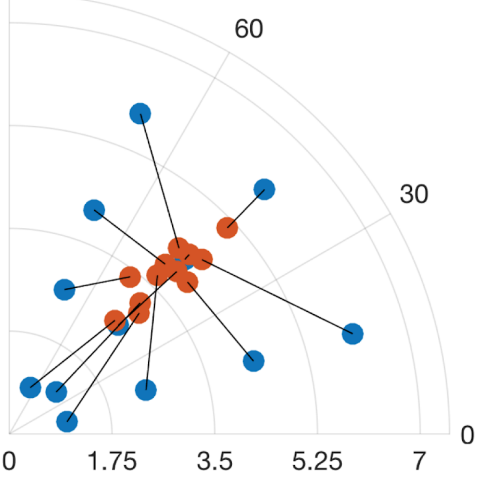

V2

60

60
90

60

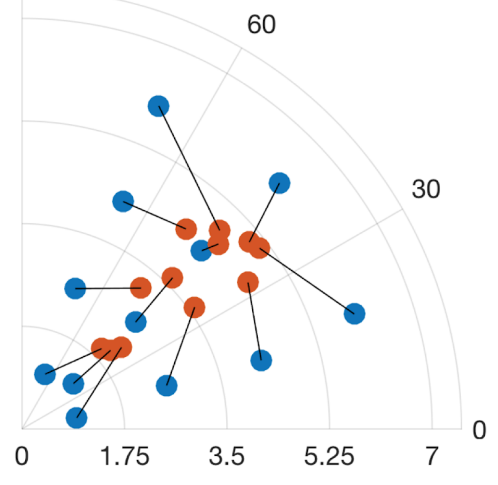

LO2

90

60

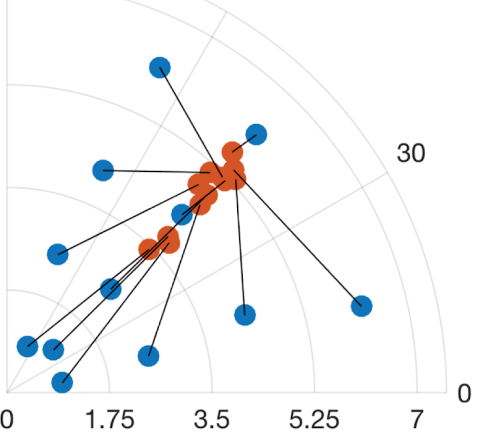

Figure S1. Changes in pRF position in response to the presentation of an AS. The figures show position changes between in figure 1. 

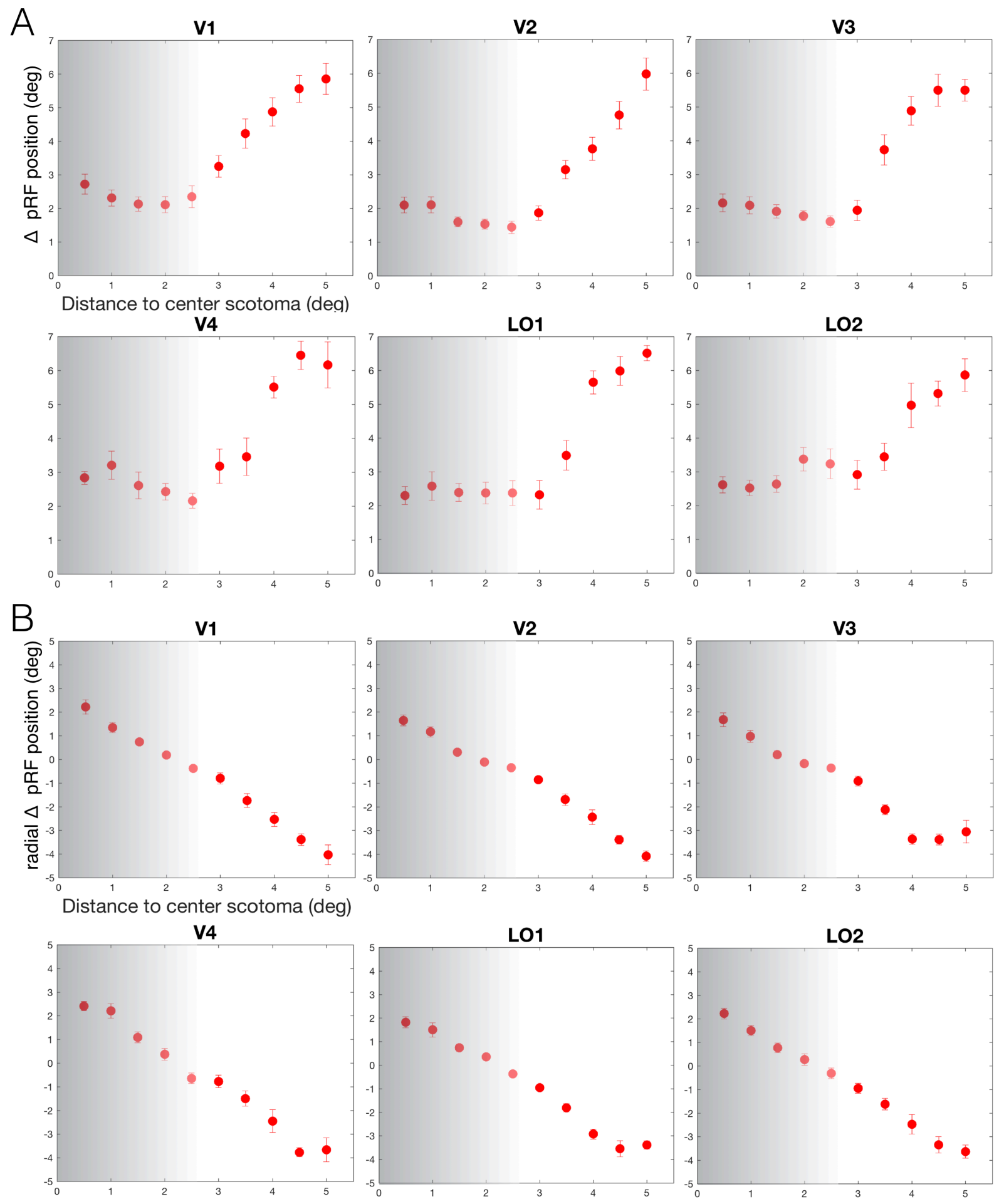

Figure S2. Change in preferred position of pRFs as a function of their distance to the AS. A: pRF position change as a function of the Euclidean distance between the pRF position and the center of the scotoma (bins of 0.5 deg) for all the visual areas analysed. The error bars represent the standard error. B: Change in radial pRF position as a function of the radial distance between the pRF position and the center of the scotoma. 


\section{Simulations of pRF shiftss}

To verify that pRF shifts did not result from a statistical bias (regression to the mean), we

580 simulated the Euclidean and radial pRF position change resulting from arbitrary shifts in

581 position. We simulated 10000 pRF positions uniformly distributed across the stimulated visual

582 field for both conditions $\left(\mathrm{AS}^{+}\right.$and $\left.\mathrm{AS}^{-}\right)$. PRF's PP were collapsed onto a single quadrant and the

583 Euclidean and radial PP shifts were binned in 0.5 degree bins as a function of the distance to the

584 center of the AS. Figure S3 shows a comparison between the simulated and measured pRF PP

585 shifts. For both types of shift (radial and Euclidean) the observed shifts cannot be explained as a

586 result of a statistical bias. Note that in panel B at the edge of the scotoma $(2.5 \mathrm{deg})$ the measured

587 position shift is $\sim 0 \mathrm{deg}$ whereas the simulated shift is $\sim 1.5 \mathrm{deg}$. Moreover the voxels located

588 near the center of the scotoma are displaced of 2.2 deg (corresponding to distance between the

589 center of the AS to its edge) while the simulated displacement is the double.
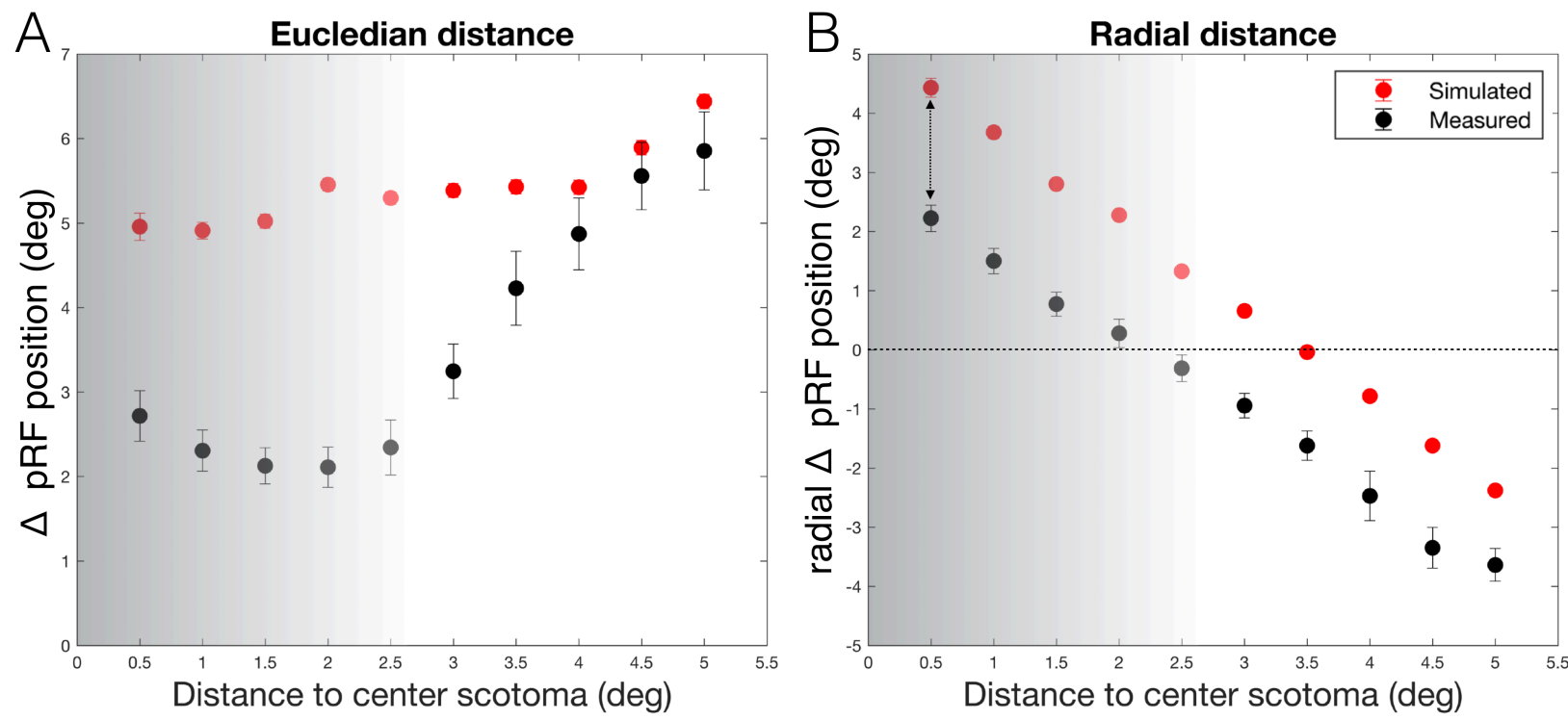

Figure S3. Simulated position change as a function of the distance to the AS. A: Simulated pRF position change as a

function of the Euclidean distance between pRF position measured with $\mathrm{AS}^{-}$and the center of the scotoma, in bins of 0.5 deg.

593 Error bars show the standard error of the mean over hemispheres. B: pRF position change as a function of the radial distance

594 between pRF position measured with $\mathrm{AS}^{-}$and the center of the scotoma. 


\section{Comparison between SF and FF model analyses}

596 Previous work has suggested that pRF shifts may result from disregarding the AS when creating

597 a model of the stimulus input that drives the pRF. In the main body of our paper, we described a

598 model that took the AS into account (scotoma field (SF)). Here, we show the effect of using a

599 full field (FF) model. The pRF position shifts between $\mathrm{AS}^{-}$and $\mathrm{AS}^{+}$conditions were present

600 when applying either of the both models. Furthermore, the presence of the artificial scotomas

601 neither reduced the BOLD amplitude nor affected the explained variance of the models.

$A$

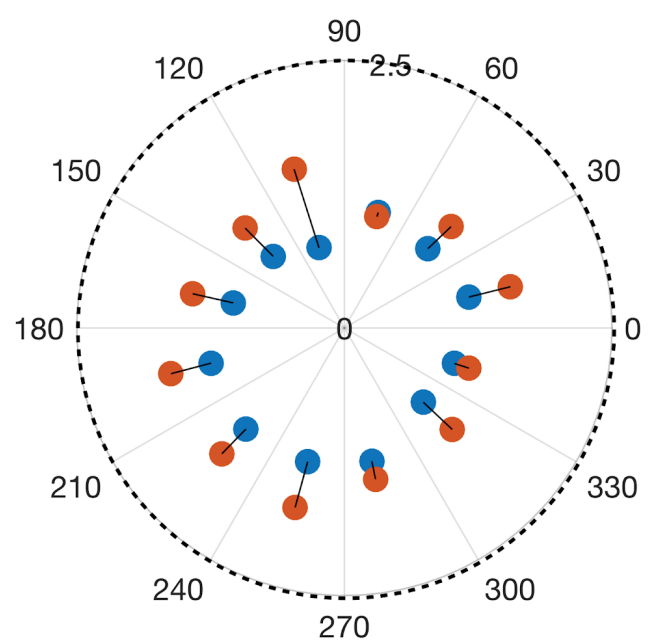

C

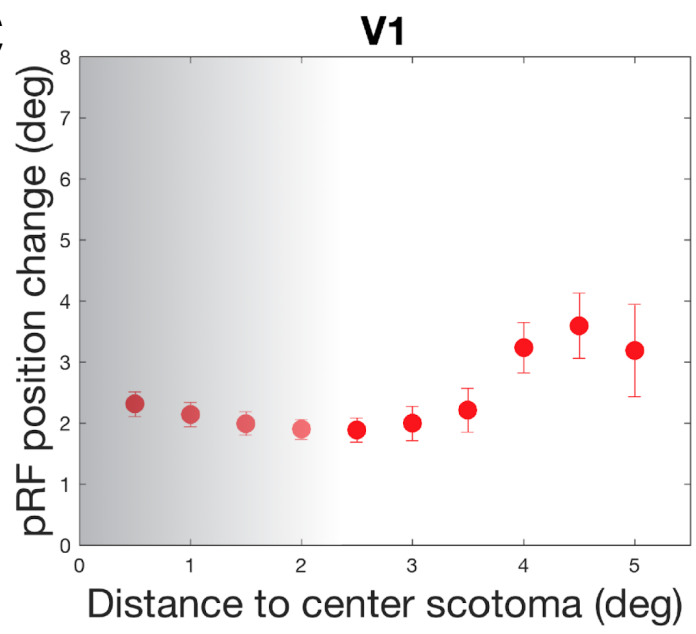

B 90

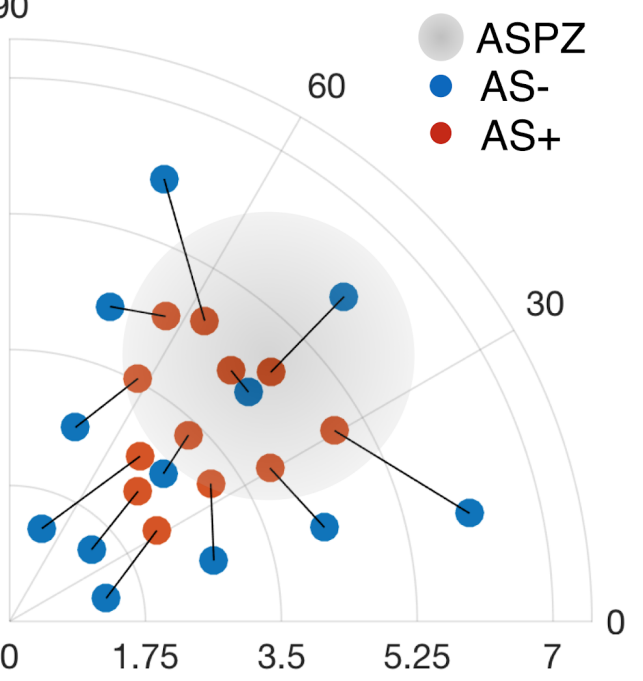

$D$

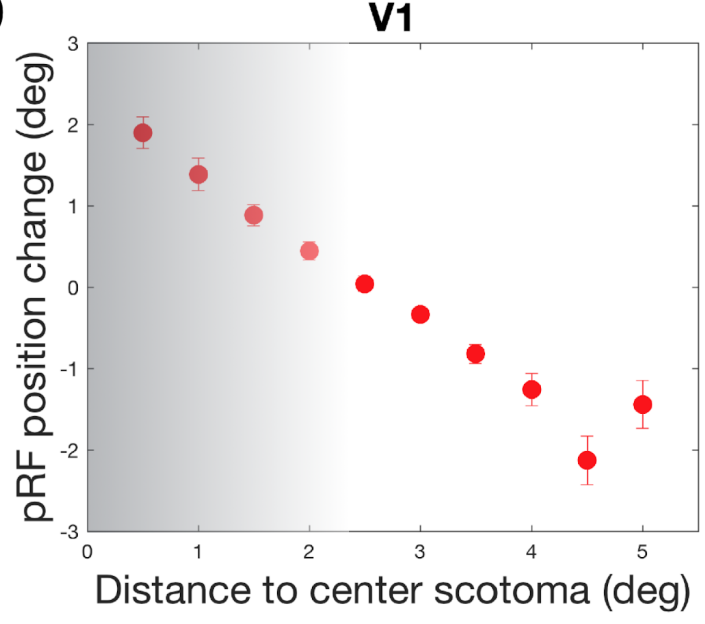


Figure S4. Changes in V1 pRF position change in response to the presentation of the AS as calculated when using a full field (FF) model. A: Shift between the two conditions $\mathrm{AS}^{-}$(blue) and $\mathrm{AS}^{+}$(red) of the pRFs with initial PPs located inside the ASPZ. B: Position change between conditions in different sectors of the visual field, averaged across participants. C: pRF position change $\left(\mathrm{AS}^{+} \mathrm{vs} \mathrm{AS}^{-}\right)$as a function of distance between pRF position (based on $\mathrm{AS}^{-}$) and the center of the scotoma (bins of $0.5 \mathrm{deg}$, Euclidean space). Error bars show the standard error of the mean across hemispheres. D: The change in radially projected pRF position change as a function of the radial distance between pRF position measured in the $\mathrm{AS}^{-}$and the center of the scotoma. The gray transparent region refers to the AS, the darker region corresponds to the center of the AS.

610
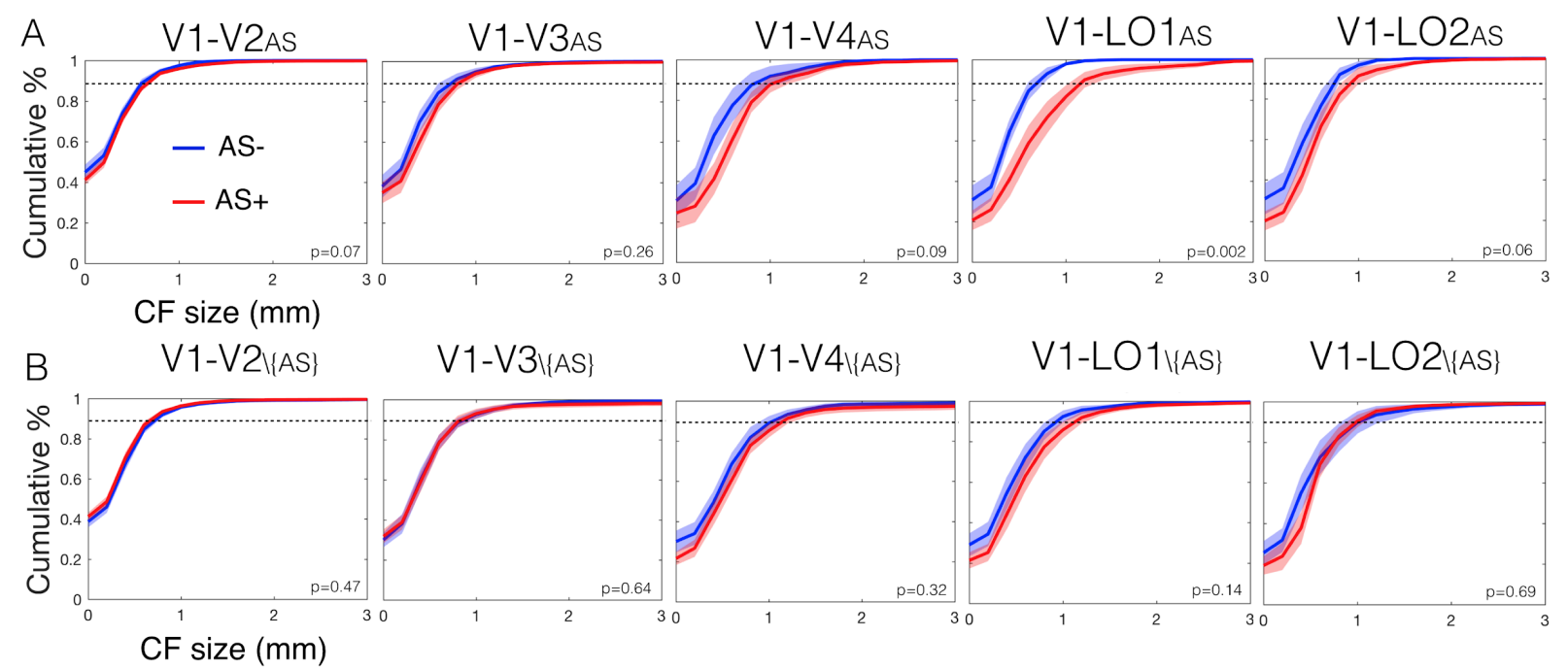

614 calculated for the voxels within the ASPZ in the target visual areas V2 a LO2. B: Analogous analysis to panel A, but for those

615 voxels outside the ASPZ. The shaded area represents the $5 \%$ and $95 \%$ confidence intervals. The p-value on the bottom right of 616 each graph shows the significance of the difference between the two conditions.

618 Six of the seven participants included in the MRI study, participated in a psychophysical 619 experiment to establish the time required for filling-in to occur. The stimulus consisted of 620 dynamic white noise band pass filtered at frequencies of 2 to $4 \mathrm{cpd}$. Four AS with a radius of 2.5 621 deg were superimposed. The participant's task was to fixate in the center of the screen 622 (represented by a white dot -0.15 deg radius) and press a button when the background was 
623 perceived as uniform (the AS had been filled in). Filling-in time corresponded to the time

624 interval since the presentations of the scotomas until the button press was recorded. The

625 scotomas were centered at $4.5 \mathrm{deg}$ eccentricity, at each quarter field. Per participant four

626 repetitions (trials) were performed. Between two consecutive trials there was a gap of 15s during

627 which a uniform grey background was shown in order to prevent carryover. The filling-in time

628 was always less than one minute (figure S6). Therefore one minute of filling-in time was allowed

629 in the fMRI experiment for all participants.

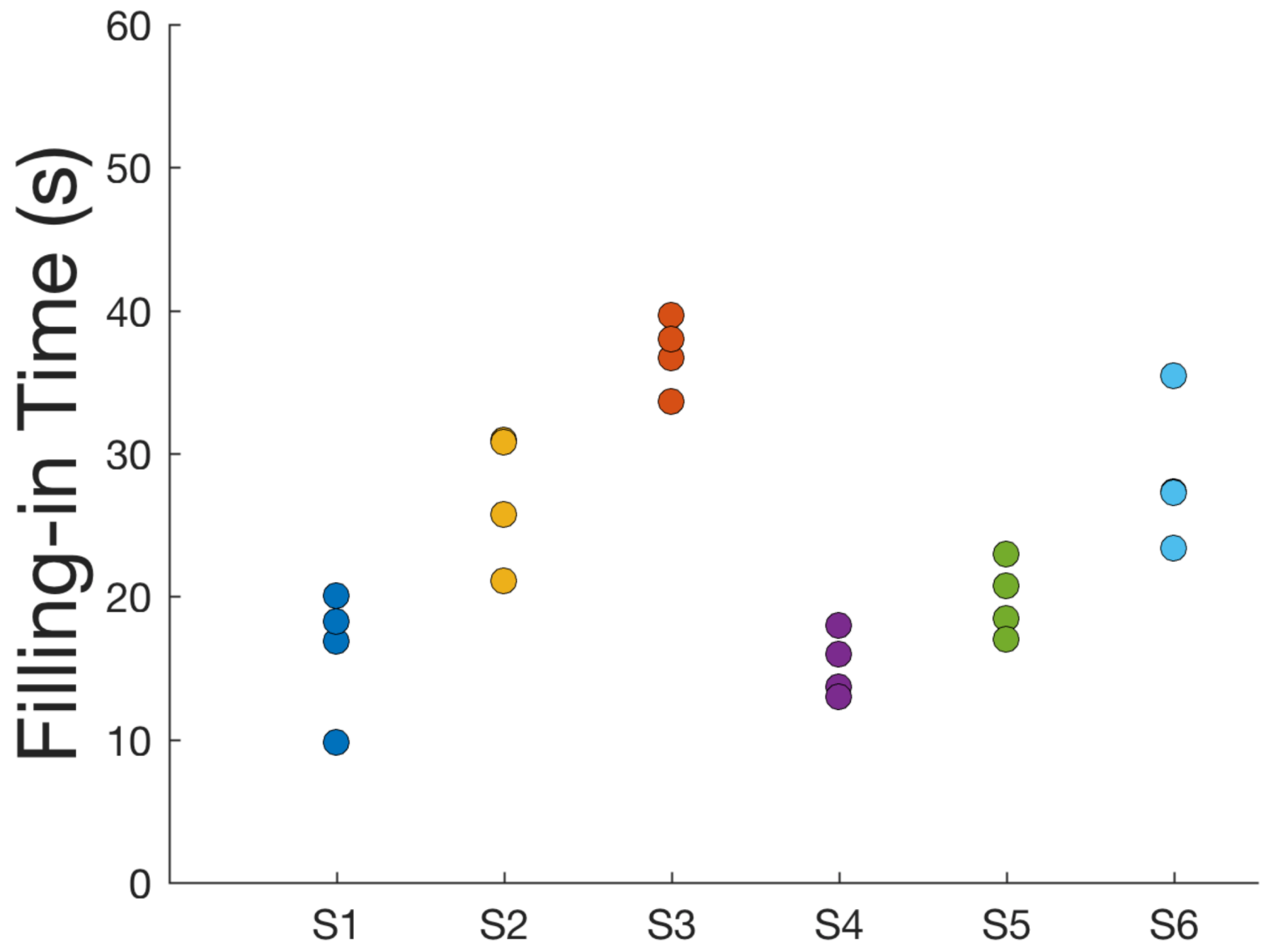

631 Figure S6. Results of the psychophysical tests used to define the optimal stimulus parameters (adaptation time). Filling-in 632 time measured per trial and per participant. 
bioRxiv preprint doi: https://doi.org/10.1101/758094; this version posted September 8, 2019. The copyright holder for this preprint (which was not certified by peer review) is the author/funder, who has granted bioRxiv a license to display the preprint in perpetuity. It is made available under aCC-BY-NC-ND 4.0 International license.

\section{6. Attention task performance}

\begin{tabular}{|l|l|l|}
\hline Task & Mean (\%) & Standard error (\%) \\
\hline LCR & 90.9 & 6.8 \\
\hline AS- & 86.0 & 8.7 \\
\hline AS+ & 87.7 & 3.4 \\
\hline
\end{tabular}

635

636 Table S1. Performance (average and standard error) of the attention task per condition. One-way repeated measures 637 ANOVA showed no significant difference between the attention task performance between the conditions $\mathrm{AS}^{+}$and $\mathrm{AS}^{-}$ $638 \quad(\mathrm{p}=0.6341)$. 